\title{
Bismuth(III) Chloride Catalyzed Intramolecular Hetero-Diels-Alder Reaction: Access to cis-Fused Angular Hexahydrobenzo[c]acridines
}

\author{
Gowravaram Sabitha* \\ Kontham Shankaraiah \\ Kancherla Sindhu \\ Bejawada Madhavi Latha
}

Natural Products Chemistry Division, CSIR-Indian Institute of

Chemical Technology, Hyderabad 500 007, India

gowravaramsr@yahoo.com

sabitha@iict.res.in

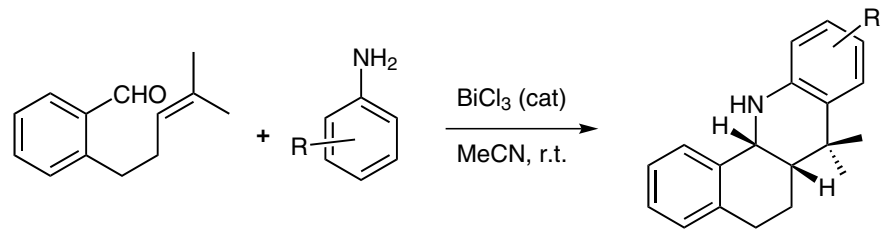

88\% yield from 2-methylbenzaldehyde and 1-bromo-3methylbut-2-ene (prenyl bromide) in the presence of $N, N, N^{\prime}$-trimethylethane-1,2-diamine ${ }^{6}$ (Scheme 1).

Published online: 06.10 .2014

DOI: 10.1055/s-0034-1379022; Art ID: ss-2014-z0258-op

Abstract New polycyclic hexahydrobenzo[c]acridines were synthesized in excellent yields by intramolecular [4+2]-cycloaddition reactions of aldimines derived from aromatic amines and 2-(4-methylpent-3-en1 -yl)benzaldehyde in acetonitrile in the presence of $10 \mathrm{~mol} \%$ of bismuth(III) chloride. The reaction is highly diastereoselective, giving cisfused benzoacridine derivatives preferentially.

Key words hetero-Diels-Alder reactions, catalysis, heterocycles, polycycles, aldimines, aldehydes

The intramolecular hetero-Diels-Alder reaction ${ }^{1,2}$ has become an attractive tool for the synthesis of complex molecular structures. Angularly fused polycyclic nitrogen heterocycles $^{3}$ are of great importance to chemists and biologists because of the significant role played by such compounds in biological systems and in medicinal chemistry. Heterocyclic systems containing the hexahydrobenzo[a]acridine skeleton can be prepared by cascade heterocyclization of cyclic diketones with aromatic amines and vanillyl esters. ${ }^{4}$ Benzoacridine derivatives have been reported to possess a significant inhibitory effect on the growth of KB human papilloma cells, ${ }^{5}$ an activity that is creating interest in further studies on such derivatives. To the best of our knowledge, there have been no reported syntheses of angularly fused hexahydrobenzoacridine derivatives from 2-(4-methylpent-3-en-1-yl)benzaldehyde (1) ${ }^{6}$ and aromatic amines. As a continuation of our work on [4+2] cycloaddition chemistry, ${ }^{7}$ and on the basis of a report in the literature, ${ }^{8}$ we have developed a novel synthesis of angularly fused benzoacridine derivatives by means of the intramolecular hetero-Diels-Alder reaction of 2-(4-methylpent-3en-1-yl)benzaldehyde with aromatic amines in the presence of bismuth(III) chloride. Aldehyde $\mathbf{1}$ was prepared in

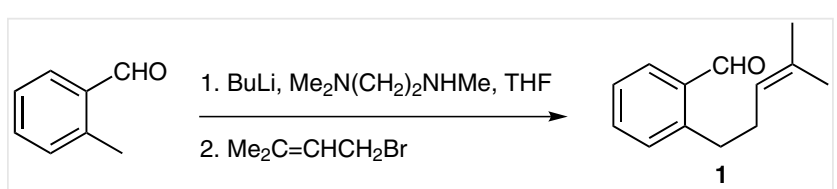

Scheme 1 Preparation of 2-(4-methylpent-3-en-1-yl)benzaldehyde (1)

The use of bismuth trichloride in the cyclization of aldehyde $\mathbf{1}$ with amines has several advantages. Bismuth is the least toxic of the heavy elements ${ }^{9 a, b}$ and the biochemistry, ${ }^{9 c}$ toxicology, ${ }^{9 \mathrm{~d}}$ and environmental effects ${ }^{9 \mathrm{e}}$ of bismuth compounds have been reviewed. Moreover, bismuth compounds are employed as catalysts in industry for the manufacture of acrolein and acrylonitrile, and they are also used in pharmaceutical products.

Having prepared aldehyde 1, we examined its intramolecular [4+2]-cycloaddition reactions. Initially, we studied the reaction of aldehyde $\mathbf{1}$ with aniline (2a) in the presence of $10 \mathrm{~mol} \%$ bismuth(III) chloride in acetonitrile at $85{ }^{\circ} \mathrm{C}$, and we obtained 7,7-dimethyl-5,6,6a,7,12,12a-hexahydrobenzo[c] acridine (3a) in $82 \%$ yield (Scheme 2 ). The reaction was complete in one hour, as indicated by thin-layer chromatography. The reaction proceeds by generation of an imine that undergoes an intramolecular hetero-Diels-Alder reaction to give the product 3a as a mixture of cis- and trans-diastereomers with the former as the predominant product. The ratio of the cis- and trans-isomers of 3a was determined by NMR spectroscopic studies on the crude product. In compound 3a, the $\mathrm{H}-6 \mathrm{a}$ proton appears as a doublet of triplets at $\delta=1.83$. This is the result of coupling of the H-6a proton with the $\mathrm{H}-6^{\prime}$ proton, resulting in a doublet with a large $J$ value $\left(J_{6 a, 6^{\prime}}=12.3 \mathrm{~Hz}\right)$ that is split into a triplet with a small $J$ 
value (3.4 Hz) by coupling with the equatorial $\mathrm{H}-12 \mathrm{a}$ and $\mathrm{H}-$ 6 protons. This corresponds to a cis-fusion at the junction. The doublet for the $\mathrm{H}-12 \mathrm{a}$ proton appears at $\delta=4.65$ with a small $J$ value as a result of vicinal coupling with an equatorial $\mathrm{H}$-6a proton $\left(J_{12 \mathrm{a}, 6 \mathrm{a}}=3.2 \mathrm{~Hz}\right)$. The cis-configuration of $\mathrm{H}$ $6 \mathrm{a}$ and $\mathrm{H}-12 \mathrm{a}$ in compound 3 was confirmed by the strong NOE peaks. Confirmation of the cis-fusion of 3a was also obtained by direct comparison with data reported in the literature, ${ }^{7 b, d, f}$ in addition to the ${ }^{1} \mathrm{H}$ NMR spectral data.

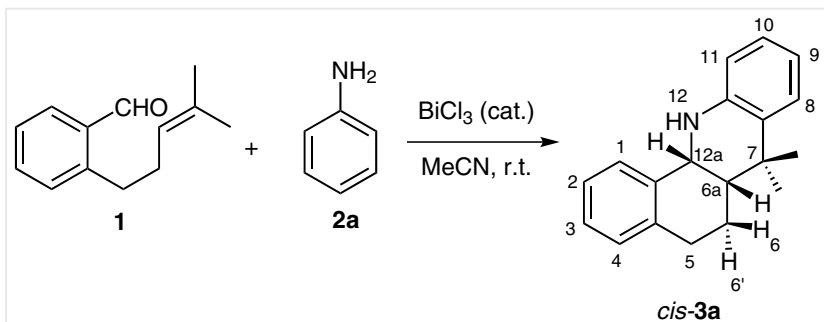

Scheme 2 Preparation of 7,7-dimethyl-5,6,6a,7,12,12a-hexahydrobenzo[c]acridine (3a)

In an effort to demonstrate the scope of the bismuth(III) chloride-catalyzed hetero-Diels-Alder reaction, we examined the reactions of aldehyde $\mathbf{1}$ with several anilines $\mathbf{2 b}-\mathbf{k}$ bearing various substituents (Table 1 ). Amines bearing electron-donating groups and those bearing electron-withdrawing groups both gave the corresponding products $\mathbf{3}$ in high yields under similar reaction conditions to those used for aniline (2a); the substituents had no obvious effect on the yield or the reaction time under optimized conditions. In all cases, the cis-annulated products were formed predominantly. The products were characterized by means of IR, ${ }^{1} \mathrm{H}$ and ${ }^{13} \mathrm{C}$ NMR, and mass spectroscopy. Acetonitrile was selected as the optimal solvent, as it gave better results than other solvents tested such as methanol, tetrahydrofuran, diethyl ether, dichloromethane, or 1,2-dichloroethane.

Mechanistically, the reaction proceeds through formation of an imine from the aromatic amine and 2-(4-methylpent-3-en-1-yl)benzaldehyde (1). The imine then undergoes a Lewis acid induced intramolecular hetero-DielsAlder reaction to give the hexahydrobenzo[c]acridine (Scheme 3).

In conclusion, we have demonstrated a convenient synthesis of cis-fused hexahydrobenzo[c]acridines derivatives in good yields in a one-pot operation using bismuth(III) chloride. The advantages of the present protocol are mild
Table 1 Synthesis of Angular Hexahydrobenzo[c]acridines 3a-k

\begin{tabular}{llllllll} 
& & & & \\
\hline
\end{tabular}

conditions, short reaction times, and the use of a soft Lewis acid catalyst that is commercially available. The method is therefore highly practical and might find wide applicability in organic synthesis.

All reagents and catalysts were purchased from Sigma-Aldrich. Reactions were conducted under $\mathrm{N}_{2}$ in anhydrous solvents such as THF or $\mathrm{MeCN}$. All reactions were monitored by TLC on Merck $60 \mathrm{~F}-254$ silica gel plates visualized under UV radiation. Hexanes (bp $60-80^{\circ} \mathrm{C}$ ) and EtOAc were used for silica gel column chromatography. Yields refer to chromatographically and spectroscopically $\left({ }^{1} \mathrm{H}\right.$ and ${ }^{13} \mathrm{C}$ NMR $)$ homogeneous materials. Air-sensitive reagents were transferred by syringe. Evaporation of solvents was performed at reduced pressure on a $\mathrm{Bu}-$ chi rotary evaporator. ${ }^{1} \mathrm{H}$ and ${ }^{13} \mathrm{C}$ NMR spectra of samples in $\mathrm{CDCl}_{3}$ were recorded on Bruker UXNMR FT-300 MHz (Avance) spectrometers with TMS as an internal standard. Mass spectra were recorded on

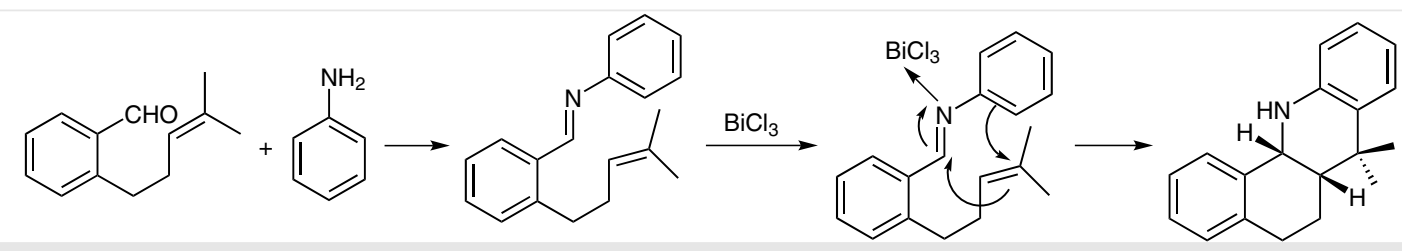

Scheme 3 A plausible reaction mechanism 
a Finnigan MAT 1020B or a Micromass VG 70-70 H spectrometer operated at $70 \mathrm{eV}$ with a direct inlet system. Column chromatography was performed on silica gel (60-20 mesh; ACME Chemicals, Mumbai).

\section{2-(4-Methylpent-3-en-1-yl)benzaldehyde (1)}

A solution of $\mathrm{Me}_{2} \mathrm{~N}\left(\mathrm{CH}_{2}\right)_{2} \mathrm{NHMe}(1.1 \mathrm{~mL}, 9.8 \mathrm{mmol}, 1.1$ equiv) in THF $(20 \mathrm{~mL})$ at $-20{ }^{\circ} \mathrm{C}$ was sequentially treated with a $1.6 \mathrm{M}$ solution of BuLi in hexanes (5.89 mL, $9.43 \mathrm{mmol}, 1.05$ equiv) and 2- $\mathrm{MeC}_{6} \mathrm{H}_{4} \mathrm{CHO}$ $(1.08 \mathrm{~g}, 8.98 \mathrm{mmol})$. After $15 \mathrm{~min}$, a further 3 equivalents of BuLi were added at $-20^{\circ} \mathrm{C}, \mathrm{Me}_{2} \mathrm{C}=\mathrm{CCH}_{2} \mathrm{Br}$ (5.4 mL, $35.95 \mathrm{mmol}, 4$ equiv) was added at $-78^{\circ} \mathrm{C}$, and the mixture was stirred for $30 \mathrm{~min}$. The resulting mixture was then poured into cold sat. aq $\mathrm{NH}_{4} \mathrm{Cl}(10 \mathrm{~mL})$ and extracted with EtOAc $(3 \times 20 \mathrm{~mL})$. The extracts were combined, washed with brine $(2 \times 10 \mathrm{~mL})$, dried $\left(\mathrm{Na}_{2} \mathrm{SO}_{4}\right)$, and concentrated under reduced pressure. The crude product was purified by flash column chromatography [silica gel, hexane-EtOAc (15:1)] to give a colorless oil; yield: $1.48 \mathrm{~g}(88 \%)$.

IR (neat): 3447, 2961, 2927, 2860, 1696, 1600, 1450, 1207, $757 \mathrm{~cm}^{-1}$.

${ }^{1} \mathrm{H}$ NMR (300 MHz, $\mathrm{CDCl}_{3}$ ): $\delta=10.27(\mathrm{~s}, 1 \mathrm{H}), 7.83$ (dd, $J=7.7,1.3 \mathrm{~Hz}, 1$ $\mathrm{H}), 7.50(\mathrm{td}, J=7.6,1.3 \mathrm{~Hz}, 1 \mathrm{H}), 7.36(\mathrm{td}, J=7.6,1.0 \mathrm{~Hz}, 1 \mathrm{H}), 7.27(\mathrm{~d}$, $J=8.2 \mathrm{~Hz}, 1 \mathrm{H}$ ), $5.20-5.15$ (m, $1 \mathrm{H}$ ), 3.08-3.01 (m, $2 \mathrm{H}), 2.30$ (qt, $J=$ 15.1, 7.5 Hz, $2 \mathrm{H}), 1.66$ (s, $3 \mathrm{H}), 1.45$ (s, $3 \mathrm{H})$.

${ }^{13} \mathrm{C}$ NMR (75 MHz, $\mathrm{CDCl}_{3}$ ): $\delta=191.9,145.0,133.6$ (2 C), 131.1, 130.9, $126.4,122.7,32.3,30.5,25.6,17.4$. .

ESI-MS: $m / z=211\left[\mathrm{M}^{+}+\mathrm{Na}\right]$.

\section{7,7-Dimethyl-5,6,6a,7,12,12a-hexahydrobenzo[c]acridines 3a-k; General Procedure}

$\mathrm{BiCl}_{3}$ (10 mol\%) was added to a mixture of the appropriate aromatic amine $\mathbf{2 a}-\mathbf{k}(1 \mathrm{mmol})$ and aldehyde $\mathbf{1}(1.1 \mathrm{mmol})$ in anhyd $\mathrm{MeCN}(5$ $\mathrm{mL}$ ), and the mixture was refluxed at $85^{\circ} \mathrm{C}$ for $1 \mathrm{~h}$. When the reaction was complete (TLC), the mixture was filtered through Celite. The filtrate was extracted with EtOAc $(3 \times 20 \mathrm{~mL})$, and the extracts were combined, washed with brine $(2 \times 10 \mathrm{~mL})$, dried $\left(\mathrm{Na}_{2} \mathrm{SO}_{4}\right)$, and concentrated. The crude product was purified by chromatography [silica gel, hexanes-EtOAc (16:1)].

\section{7,7-Dimethyl-5,6,6a,7,12,12a-hexahydrobenzo[c]acridine (3a)}

Pale yellow viscous liquid; yield: $251 \mathrm{mg}$ (82\%).

IR (neat): 3445, 3062, 2961, 2926, 1603, 1562, 1474, 1216, $758 \mathrm{~cm}^{-1}$.

${ }^{1} \mathrm{H} \mathrm{NMR}\left(300 \mathrm{MHz}, \mathrm{CDCl}_{3}\right): \delta=7.40-7.30(\mathrm{~m}, 5 \mathrm{H}), 6.67-6.61(\mathrm{~m}, 1 \mathrm{H})$, $6.37(\mathrm{~d}, J=7.7 \mathrm{~Hz}, 1 \mathrm{H}), 5.69(\mathrm{dt}, J=7.3,1.3 \mathrm{~Hz}, 1 \mathrm{H}), 4.65(\mathrm{~d}, J=3.2 \mathrm{~Hz}$, $1 \mathrm{H}), 4.29(\mathrm{~s}, 1 \mathrm{H}), 2.93-2.87(\mathrm{~m}, 1 \mathrm{H}), 2.82(\mathrm{dd}, J=12.0,6.1 \mathrm{~Hz}, 1 \mathrm{H})$, 2.68-2.64 (m, $1 \mathrm{H}), 1.83(\mathrm{dt}, J=12.3,3.4 \mathrm{~Hz}, 1 \mathrm{H}), 1.70-1.65(\mathrm{~m}, 1 \mathrm{H})$, $1.45(\mathrm{~s}, 3 \mathrm{H}), 1.36(\mathrm{~s}, 3 \mathrm{H})$.

${ }^{13} \mathrm{C}$ NMR $\left(75 \mathrm{MHz}, \mathrm{CDCl}_{3}\right): \delta=141.4,130.7,129.2,128.2,128.1,127.9$, 127.6, 126.7, 126.6, 124.9, 117.3, 112.6, 45.8, 40.5, 32.3, 28.3, 25.6, 25.0, 17.6.

ESI-MS: $m / z=265\left[\mathrm{M}^{+}+\mathrm{H}\right]$.

HRMS: $m / z[\mathrm{M}+\mathrm{H}]^{+}$calcd for $\mathrm{C}_{19} \mathrm{H}_{22} \mathrm{~N}$ : 264.1752; found: 264.1761 .

\section{7,7,9-Trimethyl-5,6,6a,7,12,12a-hexahydrobenzo[c]acridine (3b)}

Pale yellow viscous liquid; yield: $221 \mathrm{mg}$ (80\%).

IR (neat): 3449, 2924, 2854, 1634, 1459, 1217, $765 \mathrm{~cm}^{-1}$.

${ }^{1} \mathrm{H} \mathrm{NMR}\left(300 \mathrm{MHz}, \mathrm{CDCl}_{3}\right): \delta=7.42(\mathrm{~d}, J=7.6 \mathrm{~Hz}, 1 \mathrm{H}), 7.38-7.28(\mathrm{~m}, 1$ $\mathrm{H}), 7.20(\mathrm{dt}, J=7.9,0.6 \mathrm{~Hz}, 1 \mathrm{H}), 7.17-7.14(\mathrm{~m}, 1 \mathrm{H}), 7.11(\mathrm{~d}, J=1.3 \mathrm{~Hz}$, $1 \mathrm{H}), 6.85-6.83(\mathrm{~m}, 1 \mathrm{H}), 6.59(\mathrm{~d}, J=7.9 \mathrm{~Hz}, 1 \mathrm{H}), 4.30(\mathrm{~d}, J=10.3 \mathrm{~Hz}, 1$
H), 4.22-4.11 (br s, $1 \mathrm{H}), 2.93-2.89(\mathrm{~m}, 1 \mathrm{H}), 2.27$ (s, $3 \mathrm{H}), 2.08-2.03$ (m, $1 \mathrm{H}), 1.79-1.73(\mathrm{~m}, 1 \mathrm{H}), 1.59-1.50(\mathrm{~m}, 2 \mathrm{H}), 1.40(\mathrm{~s}, 3 \mathrm{H}), 1.23$ (s, $3 \mathrm{H})$.

${ }^{13} \mathrm{C}$ NMR $\left(75 \mathrm{MHz}, \mathrm{CDCl}_{3}\right): \delta=137.7,135.7,132.2,130.5,129.1,128.3$, 128.2, 127.3, 126.6, 126.3, 125.7, 116.0, 51.4, 46.9, 35.9, 30.9, 28.3, 27.9, 21.9, 17.6.

ESI-MS: $m / z=278\left[\mathrm{M}^{+}+\mathrm{H}\right]$.

HRMS: $m / z[\mathrm{M}+\mathrm{H}]^{+}$calcd for $\mathrm{C}_{20} \mathrm{H}_{24} \mathrm{~N}$ : 278.1908; found: 278.1902 .

9-Methoxy-7,7-dimethyl-5,6,6a,7,12,12a-hexahydrobenzo[c]acridine (3c)

Colorless viscous liquid; yield: $249 \mathrm{mg}$ (85\%).

IR (neat): 3423, 3063, 2959, 2933, 1612, 1512, 1474, 1228, 1037, 819 $\mathrm{cm}^{-1}$.

${ }^{1} \mathrm{H}$ NMR $\left(300 \mathrm{MHz}, \mathrm{CDCl}_{3}\right): \delta=7.38-7.13(\mathrm{~m}, 4 \mathrm{H}), 6.84-6.77(\mathrm{~m}, 1 \mathrm{H})$, 6.64-6.55 (m, $1 \mathrm{H}), 6.34(\mathrm{~d}, J=8.3 \mathrm{~Hz}, 1 \mathrm{H}), 4.60(\mathrm{~d}, J=3.0 \mathrm{~Hz}, 1 \mathrm{H})$, $4.24(\mathrm{~s}, 1 \mathrm{H}), 3.75(\mathrm{~s}, 3 \mathrm{H}), 2.91-2.79(\mathrm{~m}, 1 \mathrm{H}), 2.68(\mathrm{dd}, J=15.1,7.5 \mathrm{~Hz}$, $1 \mathrm{H}), 1.87-1.78(\mathrm{~m}, 1 \mathrm{H}), 1.71-1.46(\mathrm{~m}, 2 \mathrm{H}), 1.43(\mathrm{~m}, 3 \mathrm{H}), 1.37(\mathrm{~m}, 3$ $\mathrm{H})$.

${ }^{13} \mathrm{C}$ NMR $\left(75 \mathrm{MHz}, \mathrm{CDCl}_{3}\right): \delta=159.4,141.0,137.9,130.4,129.3,128.1$, $126.6,126.5,126.0,114.8,111.7,111.3,70.8,55.7,55.3,40.8,28.3$, 25.6, 25.1, 17.6. 6 .

ESI-MS: $m / z=294\left[\mathrm{M}^{+}+\mathrm{H}\right]$.

HRMS: $m / z[\mathrm{M}+\mathrm{H}]^{+}$calcd for $\mathrm{C}_{20} \mathrm{H}_{24} \mathrm{NO}$ : 294.1857; found: 294.1859 .

11-Chloro-7,7-dimethyl-5,6,6a,7,12,12a-hexahydrobenzo[c]acridine (3d)

Pale yellow viscous liquid; yield: $243 \mathrm{mg}$ (82\%).

IR (neat): 3436, 3063, 2961, 2929, 2869, 1696, 1496, 1303, 1240, 771, $731 \mathrm{~cm}^{-1}$.

${ }^{1} \mathrm{H}$ NMR $\left(300 \mathrm{MHz}, \mathrm{CDCl}_{3}\right): \delta=7.48(\mathrm{~d}, J=7.7 \mathrm{~Hz}, 1 \mathrm{H}), 7.33-7.27(\mathrm{~m}, 1$ H), 7.24-7.20 (m, $2 \mathrm{H}), 7.17(\mathrm{~d}, J=7.5 \mathrm{~Hz}, 1 \mathrm{H}), 7.12(\mathrm{dd}, J=7.7,1.2 \mathrm{~Hz}$, $1 \mathrm{H}), 6.6(\mathrm{t}, J=7.7 \mathrm{~Hz}, 1 \mathrm{H}), 5.01-4.96(\mathrm{br} \mathrm{s}, 1 \mathrm{H}), 4.36(\mathrm{~d}, J=10.5 \mathrm{~Hz}, 1$ H), 2.73 (dd, $J=7.7,2.8 \mathrm{~Hz}, 2 \mathrm{H}), 2.09-2.03(\mathrm{~m}, 1 \mathrm{H}), 1.80-1.74(\mathrm{~m}, 1$ $\mathrm{H}), 1.62-1.55(\mathrm{~m}, 1 \mathrm{H}), 1.41(\mathrm{~s}, 3 \mathrm{H}), 1.23(\mathrm{~s}, 3 \mathrm{H})$.

${ }^{13} \mathrm{C} \mathrm{NMR}\left(75 \mathrm{MHz}, \mathrm{CDCl}_{3}\right): \delta=136.8,131.2,129.2,128.1,126.8,126.5$, 125.5 (2 C), 125.0, 123.5, 120.8, 117.3, 51.0, 46.0, 30.3, 27.6, 26.9, 21.7, 17.8

ESI-MS: $m / z=298\left[\mathrm{M}^{+}+\mathrm{H}\right]$.

HRMS: $m / z[\mathrm{M}+\mathrm{H}]^{+}$calcd for $\mathrm{C}_{19} \mathrm{H}_{21} \mathrm{ClN}$ : 298.1362; found: 298.1354 .

9-Fluoro-7,7-dimethyl-5,6,6a,7,12,12a-hexahydrobenzo[c]acridine (3e)

Colorless viscous liquid; yield: $210 \mathrm{mg}$ (75\%).

IR (neat): 3420, 2925, 2854, 1615, 1470, 1275, 1187, $769 \mathrm{~cm}^{-1}$. ${ }^{1} \mathrm{H}$ NMR $\left(300 \mathrm{MHz}, \mathrm{CDCl}_{3}\right): \delta=7.44(\mathrm{~d}, J=7.5 \mathrm{~Hz}, 1 \mathrm{H}), 7.27-7.14(\mathrm{~m}, 3$ H), $7.00(\mathrm{dd}, J=10.6,3.0 \mathrm{~Hz}, 1 \mathrm{H}), 6.75(\mathrm{dt}, J=8.3,2.2 \mathrm{~Hz}, 1 \mathrm{H}), 6.61$ (dd, $J=8.3,5.3 \mathrm{~Hz}, 1 \mathrm{H}), 4.29(\mathrm{~d}, J=10.6 \mathrm{~Hz}, 1 \mathrm{H}), 4.29-4.26(\mathrm{br} \mathrm{s}, 1 \mathrm{H})$, $2.92(\mathrm{dd}, J=8.3,3.0 \mathrm{~Hz}, 2 \mathrm{H}), 2.09-2.01(\mathrm{~m}, 1 \mathrm{H}), 1.77-1.65(\mathrm{~m}, 1 \mathrm{H})$, $1.62-1.53(\mathrm{~m}, 1 \mathrm{H}), 1.39(\mathrm{~s}, 3 \mathrm{H}), 1.23(\mathrm{~s}, 3 \mathrm{H})$.

${ }^{13} \mathrm{C}$ NMR $\left(125 \mathrm{MHz}, \mathrm{CDCl}_{3}\right): \delta=141.2,139.3,130.7,129.8,129.7$, 128.2, 126.7, 126.6, 114.2, 114.0, 112.3, 112.1, 70.5, 40.9, 29.7, 28.3, $25.5,25.0,17.6$.

ESI-MS: $m / z=282\left[\mathrm{M}^{+}+\mathrm{H}\right]$.

HRMS: $m / z[\mathrm{M}+\mathrm{H}]^{+}$calcd for $\mathrm{C}_{19} \mathrm{H}_{21} \mathrm{FN}$ : 282.1658; found: 282.1668. 
7,7-Dimethyl-5,6,6a,7,12,12a-hexahydrobenzo[c]acridin-11-ol (3f) Colorless liquid; yield: $214 \mathrm{mg}$ (77\%).

IR (neat): 3422, 3066, 2966, 2928, 1617, 1452, 1216, $751 \mathrm{~cm}^{-1}$. ${ }^{1} \mathrm{H}$ NMR (300 MHz, $\left.\mathrm{CDCl}_{3}\right)$ : $\delta=7.42-7.30(\mathrm{~m}, 2 \mathrm{H}), 7.28-7.19(\mathrm{~m}, 3 \mathrm{H})$, 7.19-7.12 (m, $1 \mathrm{H}), 6.95-6.91$ ( $\mathrm{m}, 1 \mathrm{H}), 6.70-6.42$ (br s, $1 \mathrm{H}), 4.81-$ $4.46(\mathrm{~m}, 2 \mathrm{H}), 2.91(\mathrm{dd}, J=17.0,5.1 \mathrm{~Hz}, 1 \mathrm{H}), 2.86-2.79(\mathrm{~m}, 1 \mathrm{H}), 2.32-$ 1.99 (m, $1 \mathrm{H}), 1.88-1.81(\mathrm{~m}, 1 \mathrm{H}), 1.74-1.59$ (m, $1 \mathrm{H}), 1.40(\mathrm{~s}, 3 \mathrm{H})$, $1.36(\mathrm{~s}, 3 \mathrm{H})$.

${ }^{13} \mathrm{C} \mathrm{NMR}\left(75 \mathrm{MHz}, \mathrm{CDCl}_{3}\right): \delta=137.2,130.9,129.3,129.1,129.0,128.4$, 127.7, 126.6, 126.5, 126.0, 116.0, 113.1, 71.4, 40.7, 29.3, 28.2, 25.5, 25.1, 17.4 .

ESI-MS: $m / z=280\left[\mathrm{M}^{+}+\mathrm{H}\right]$.

HRMS: $m / z[M+H]^{+}$calcd for $\mathrm{C}_{19} \mathrm{H}_{22} \mathrm{NO}$ : 280.1701; found: 282.1707 .

\section{7,7-Dimethyl-9-nitro-5,6,6a,7,12,12a-hexahydrobenzo[c]acridine} (3g)

Yellow viscous liquid; yield: $228 \mathrm{mg}(74 \%)$.

IR (KBr): 3402, 2953, 2865, 1603, 1510, 1318, 1279, $737 \mathrm{~cm}^{-1}$

${ }^{1} \mathrm{H} \mathrm{NMR}\left(300 \mathrm{MHz}, \mathrm{CDCl}_{3}\right): \delta=8.12(\mathrm{~d}, J=2.3 \mathrm{~Hz}, 1 \mathrm{H}), 7.91(\mathrm{dt}, J=9.1$, $2.3 \mathrm{~Hz}, 1 \mathrm{H}), 7.33-7.23(\mathrm{~m}, 4 \mathrm{H}), 6.30(\mathrm{~d}, J=9.1 \mathrm{~Hz}, 1 \mathrm{H}), 4.74(\mathrm{~d}, J=3.8$ $\mathrm{Hz}, 1 \mathrm{H}), 4.55-4.50$ (br s, $1 \mathrm{H}), 2.98-2.91(\mathrm{~m}, 1 \mathrm{H}), 2.89-2.84(\mathrm{~m}, 1 \mathrm{H})$, 1.91-1.79 (m, $1 \mathrm{H}), 1.78-1.61(\mathrm{~m}, 1 \mathrm{H}), 1.64-1.56(\mathrm{~m}, 1 \mathrm{H}), 1.53(\mathrm{~s}, 3$ $\mathrm{H}), 1.35(\mathrm{~s}, 3 \mathrm{H})$.

${ }^{13} \mathrm{C} \mathrm{NMR}\left(75 \mathrm{MHz}, \mathrm{CDCl}_{3}\right): \delta=147.6,137.6,136.8,136.2,129.3,128.8$, 128.3, 126.7, 126.5, 124.2, 123.0, 111.8, 50.2, 41.9, 35.4, 32.4, 29.0, 25.4, 19.2 .

ESI-MS: $m / z=309\left[\mathrm{M}^{+}+\mathrm{H}\right]$.

HRMS: $m / z$ [M+H] $]^{+}$calcd for $\mathrm{C}_{19} \mathrm{H}_{21} \mathrm{~N}_{2} \mathrm{O}_{2}$ : 309.1603; found: 309.1595 .

\section{8,11-Dibromo-7,7-dimethyl-5,6,6a,7,12,12a-hexahydroben-} zo[c]acridine ( $3 \mathrm{~h}$ )

Brown viscous liquid; yield: $359 \mathrm{mg}(86 \%)$.

IR (neat): 3414, 3015, 2960, 2927, 1577, 1483, 1442, 1292, $742 \mathrm{~cm}^{-1}$.

${ }^{1} \mathrm{H}$ NMR $\left(300 \mathrm{MHz}, \mathrm{CDCl}_{3}\right): \delta=7.33-7.22(\mathrm{~m}, 3 \mathrm{H}), 7.18(\mathrm{~d}, J=7.3 \mathrm{~Hz}, 1$ H), $7.04(\mathrm{~d}, J=8.4 \mathrm{~Hz}, 1 \mathrm{H}), 6.75(\mathrm{~d}, J=8.4 \mathrm{~Hz}, 1 \mathrm{H}), 4.60(\mathrm{~d}, J=2.7 \mathrm{~Hz}$, $1 \mathrm{H}$ ), 4.53-4.47 (br s, $1 \mathrm{H}$ ), 2.95-2.90 (m, $1 \mathrm{H}), 2.83$ (dd, $J=11.7,6.2$ $\mathrm{Hz}, 1 \mathrm{H}), 1.97-1.90$ (m, $1 \mathrm{H}), 1.80$ (s, $3 \mathrm{H}), 1.69-1.64$ (m, $1 \mathrm{H}), 1.58$ (dd, $J=12.6,5.9 \mathrm{~Hz}, 1 \mathrm{H}), 1.53(\mathrm{~s}, 3 \mathrm{H})$.

${ }^{13} \mathrm{C}$ NMR $\left(125 \mathrm{MHz}, \mathrm{CDCl}_{3}\right): \delta=41.5,137.1,136.9,130.6,129.2,129.0$, 128.1, 127.2, 126.3, 123.6, 122.2, 107.7, 49.8, 46.8, 37.9, 29.8, 29.3, 27.4, 18.9.

ESI-MS: $m / z=422[\mathrm{M}+2]^{+}, 424[\mathrm{M}+4]^{+}$.

HRMS: $m / z[\mathrm{M}+\mathrm{H}]^{+}$calcd for $\mathrm{C}_{19} \mathrm{H}_{20} \mathrm{Br}_{2} \mathrm{~N}$ : 419.9962; found: 419.9954 .

\section{8,10-Dibromo-7,7-dimethyl-5,6,6a,7,12,12a-hexahydroben-} zo[c]acridine (3i)

Brown viscous liquid; yield: $342 \mathrm{mg}(82 \%)$.

IR (neat): 3412, 3015, 2961, 2927, 1577, 1443, 1293, $755 \mathrm{~cm}^{-1}$.

${ }^{1} \mathrm{H}$ NMR $\left(300 \mathrm{MHz}, \mathrm{CDCl}_{3}\right): \delta=7.28-7.25(\mathrm{~m}, 1 \mathrm{H}), 7.24-7.22(\mathrm{~m}, 2 \mathrm{H})$, $7.17(\mathrm{~d}, J=7.3 \mathrm{~Hz}, 1 \mathrm{H}), 7.00(\mathrm{~d}, J=2.0 \mathrm{~Hz}, 1 \mathrm{H}), 6.45(\mathrm{~d}, J=2.0 \mathrm{~Hz}, 1 \mathrm{H})$, $4.54(\mathrm{~d}, J=2.3 \mathrm{~Hz}, 1 \mathrm{H}), 3.92-3.86(\mathrm{br} \mathrm{s}, 1 \mathrm{H}), 2.95-2.90(\mathrm{~m}, 1 \mathrm{H}), 2.83$ (dd, $J=11.7,6.8 \mathrm{~Hz}, 1 \mathrm{H}), 1.96-1.90(\mathrm{~m}, 1 \mathrm{H}), 1.77(\mathrm{~s}, 3 \mathrm{H}), 1.63-1.54$ (m, $2 \mathrm{H}), 1.49$ (s, $3 \mathrm{H})$.

${ }^{13} \mathrm{C}$ NMR $\left(125 \mathrm{MHz}, \mathrm{CDCl}_{3}\right): \delta=145.6,137.0,136.2,130.9,129.2$, $128.9,128.1,126.3,125.4,119.9,115.5,114.0,49.3,46.5,37.2,29.3$, 29.6, 27.6, 19.0.
ESI-MS: $m / z=422[\mathrm{M}+2]^{+}, 424[\mathrm{M}+4]^{+}$.

HRMS: $m / z[\mathrm{M}+\mathrm{H}]^{+}$calcd for $\mathrm{C}_{19} \mathrm{H}_{20} \mathrm{Br}_{2} \mathrm{~N}$ : 419.9962; found: 419.9976 .

9-Methoxy-7,7-dimethyl-5,6,6a,7,12,12a-hexahydrobenzo[c]acridine-11-carboxylic Acid (3j)

Colorless gummy liquid; yield: $293 \mathrm{mg}$ (87\%).

IR (KBr): 3450, 2960, 1652, 1583, 1497, 1426, $1217 \mathrm{~cm}^{-1}$.

${ }^{1} \mathrm{H}$ NMR $\left(300 \mathrm{MHz}, \mathrm{CDCl}_{3}\right): \delta=7.43(\mathrm{~d}, J=3.0 \mathrm{~Hz}, 1 \mathrm{H}), 7.34-7.12(\mathrm{~m}, 3$ H), 7.08-7.01 (m, $1 \mathrm{H}), 6.59(\mathrm{~d}, J=9.0 \mathrm{~Hz}, 1 \mathrm{H}), 4.75(\mathrm{~d}, J=3.0 \mathrm{~Hz}, 1 \mathrm{H})$, $4.42(\mathrm{~s}, 1 \mathrm{H}), 3.77(\mathrm{~s}, 3 \mathrm{H}), 2.92-2.77(\mathrm{~m}, 1 \mathrm{H}), 2.73-2.61(\mathrm{~m}, 1 \mathrm{H})$, 2.31-2.00 (m, $1 \mathrm{H}), 1.75-1.57(\mathrm{~m}, 2 \mathrm{H}), 1.46(\mathrm{~s}, 3 \mathrm{H}), 1.34(\mathrm{~s}, 3 \mathrm{H})$.

${ }^{13} \mathrm{C}$ NMR $\left(75 \mathrm{MHz}, \mathrm{CDCl}_{3}\right): \delta=173.2,149.3,146.9,135.8,129.3,129.1$, 127.6, 127.2, 126.1, 124.8, 122.2, 114.4, 113.3, 55.8, 49.4, 44.9, 32.4, $31.8,30.5,22.5,19.3$.

ESI-MS: $m / z=338\left[\mathrm{M}^{+}+\mathrm{H}\right]$.

HRMS: $m / z[\mathrm{M}+\mathrm{H}]^{+}$calcd for $\mathrm{C}_{21} \mathrm{H}_{24} \mathrm{NO}_{3}$ : 338.1756; found: 338.1761 .

8,9,10-Trimethoxy-7,7-dimethyl-5,6,6a,7,12,12a-hexahydrobenzo[c]acridine (3k)

Colorless gummy liquid; yield: $282 \mathrm{mg}$ (80\%).

IR (neat): 3387, 2962, 2932, 1605, 1481, 1456, 1106, $752 \mathrm{~cm}^{-1}$.

${ }^{1} \mathrm{H}$ NMR $\left(300 \mathrm{MHz}, \mathrm{CDCl}_{3}\right): \delta=7.25-7.15(\mathrm{~m}, 4 \mathrm{H}), 5.76(\mathrm{~s}, 1 \mathrm{H}), 4.49$ (d, $J=2.6 \mathrm{~Hz}, 1 \mathrm{H}), 3.92(\mathrm{~s}, 3 \mathrm{H}), 3.77(\mathrm{~s}, 3 \mathrm{H}), 3.72(\mathrm{~s}, 3 \mathrm{H}), 3.59-3.53$ (br s, $1 \mathrm{H}), 2.98-2.91(\mathrm{~m}, 1 \mathrm{H}), 2.87-2.79(\mathrm{~m}, 1 \mathrm{H}), 1.96-1.90(\mathrm{~m}, 1 \mathrm{H})$, 1.73-1.64 (m, $1 \mathrm{H}), 1.57(\mathrm{~m}, 4 \mathrm{H}), 1.42(\mathrm{~s}, 3 \mathrm{H})$.

${ }^{13} \mathrm{C} \mathrm{NMR}\left(75 \mathrm{MHz}, \mathrm{CDCl}_{3}\right): \delta=156.7,154.4,152.0,138.1,137.2,132.9$, 129.1, 128.9 (2 C), 127.7, 125.9, 93.1, 60.6, 60.5, 55.5, 50.1, 46.1, 32.3, 29.4, 27.1, 24.6, 19.1 .

ESI-MS: $m / z=354\left[\mathrm{M}^{+}+\mathrm{H}\right]$.

HRMS: $m / z$ [M $+\mathrm{H}]^{+}$calcd for $\mathrm{C}_{22} \mathrm{H}_{28} \mathrm{NO}_{3}$ : 354.2069; found: 354.2058 .

\section{Acknowledgment}

K.S. thanks CSIR, New Delhi, for the award of a fellowship.

\section{Supporting Information}

Supporting information for this article is available online at http://dx.doi.org/10.1055/s-0034-1379022.

\section{References}

(1) (a) Kappe, C. O.; Murphree, S. S.; Padwa, A. Tetrahedron 1997, 53, 14179. (b) Carruthers, W. Cycloaddition Reactions in Organic Synthesis; Pergamon: Oxford, 1990. (c) Oppolzer, W. In Comprehensive Organic Synthesis; Vol. 5; Trost, B. M.; Fleming, I., Eds.; Pergamon: Oxford, 1991, 315. (d) Pindur, U.; Lutz, G.; Otto, C. Chem. Rev. 1993, 93, 741. (e) Waldmann, H. Synthesis 1994, 535. (f) Lipshutz, B. H. Chem. Rev. 1986, 86, 795. (g) Tietze, L. F.; Brasche, G.; Gericke, K. M. Domino Reaction in Organic Synthesis; Wiley-VCH: Weinheim, 2006, 296-303. (h) Tietze, L. F. Chem. Rev. 1996, 96, 115.

(2) For reviews, see: (a) Boger, D. L.; Weinreb, S. M. Hetero DielsAlder Methodology in Organic Synthesis; Academic Press: Orlando, 1987, Chaps. 2 and 9. (b) Weinreb, S. M. In Comprehensive Organic Synthesis; Vol. 5; Trost, B. M.; Fleming, I., Eds.; Per- 
gamon: Oxford, 1991, Chap. 4.2, 401. (c) Boger, D. L. In Comprehensive Organic Synthesis; Vol. 5; Trost, B. M.; Fleming, I., Eds.; Pergamon: Oxford, 1991, Chap. 4.3, 451. (d) Tietze, L. F.; Kettschau, G. Top. Curr. Chem. 1997, 189, 1. .

(3) (a) Haung, P.; Isayan, K.; Sarkissian, A.; Oh, T. J. Org. Chem. 1998, 63, 4500. (b) Yao, S.; Johannsen, M.; Hazell, R. G.; Jørgensen, K. A. Angew. Chem. Int. Ed. 1998, 37, 3121. (c) Motorina, I. A.; Grierson, D. S. Tetrahedron Lett. 1999, 40, 7211. (d) Motorina, I. A.; Grierson, D. S. Tetrahedron Lett. 1999, 40, 7215. (e) Boruah, R. C.; Ahmed, S.; Sharma, U.; Sandhu, J. S. J. Org. Chem. 2000, 65, 922.

(4) (a) Kozlov, N. G.; Basalaeva, L. I.; Dikusar, E. A. Chem. Nat. Compd. 2004, 40, 79. (b) Kozlov, N. G.; Basalaeva, L. I. Russ. J. Gen. Chem. (Engl. Transl.) 2005, 75, 617. (c) Kozlov, N. G.; Tereshko, A. B.; Gusak, K. N. Russ. J. Org. Chem. (Engl. Transl.) 2006, 42, 266. (d) Kozlov, N. G.; Tarasevich, V. A.; Vasilevskii, D. A.; Basalaeva, L. I. Russ. J. Org. Chem. (Engl. Transl.) 2006, 42, 107. (e) Kozlov, N. G.; Basalaeva, L. I.; Dikusar, E. A. Russ. J. Org. Chem. (Engl. Transl.) 2005, 41, 1637.

(5) Cholewiński, G.; Dzierzbicka, K.; Kołodziejczyk, A. M. Pharmacol. Rep. 2011, 63, 305.

(6) Denmark, S. E.; Kesler, B. S.; Moon, Y.-C. J. Org. Chem. 1992, 57, 4912.
(7) (a) Sabitha, G.; Maruthi, Ch.; Reddy, E. V.; Reddy, Ch. S.; Yadav, J. S.; Dutta, S. K.; Kunwar, A. C. Helv. Chim. Acta 2006, 89, 2728. (b) Sabitha, G.; Reddy, Ch. S.; Maruthi, Ch.; Reddy, E. V.; Yadav, J. S. Synth. Commun. 2003, 33, 3063. (c) Sabitha, G.; Reddy, E. V.; Yadav, J. S.; Ramakrishna, K. V. S.; Sankar, A. R. Tetrahedron Lett. 2002, 43, 4029. (d) Sabitha, G.; Reddy, E. V.; Maruthi, Ch.; Yadav, J. S. Tetrahedron Lett. 2002, 43, 1573. (e) Sabitha, G.; Reddy, E. V.; Yadav, J. S. Synthesis 2002, 409. (f) Sabitha, G.; Reddy, E. V.; Yadav, J. S. Synthesis 2001, 1979.

(8) Manian, R. D. R. S.; Jayashankaran, J.; Raghunathan, R. Tetrahedron Lett. 2007, 48, 4139.

(9) (a) Sax, N. I.; Lewis, R. J. Dangerous Properties of Industrial Materials, 7th ed., Vol. (i); Van Nostrand Reinhold: New York, 1989, 283. (b) Sax, N. I.; Lewis, R. J. Dangerous Properties of Industrial Materials, 7th ed., Vol. (ii); Van Nostrand Reinhold: New York, 1989, 522. (c) Dill, K.; McGown, E. L. In The Chemistry of Organic Arsenic, Antimony and Bismuth Compounds; Patai, S., Ed.; Wiley: New York, 1994, 695. (d) Wormser, U.; Nir, I. In The Chemistry of Organic Arsenic, Antimony and Bismuth Compounds; Patai, S., Ed.; Wiley: New York, 1994, 715. (e) Maeda, S. In The Chemistry of Organic Arsenic, Antimony and Bismuth Compounds; Patai, S., Ed.; Wiley: New York, 1994, 725.

This article differs from the e-first online version only in its layout; no content has been changed. 